January 2003

\title{
Antipyretic, parasitologic, and immunologic effects of combining sulfadoxine/pyrimethamine with chloroquine or paracetamol for treating uncomplicated Plasmodium falciparum malaria
}

\author{
E. Hugosson
}

Karolinska Institutet

D. Tarimo

Muhimbili University College of Health Sciences

M. Troye-Blomberg

Stockholm University

S.M. Montgomery

Karolinska Institutet

Zul Premji

Aga Khan University, zul.premji@aku.edu

See next page for additional authors

Follow this and additional works at: http://ecommons.aku.edu/eastafrica_fhs_mc_pathol

Part of the Pathology Commons

\section{Recommended Citation}

Hugosson, E., Tarimo, D., Troye-Blomberg, M., Montgomery, S., Premji, Z., Bjorkman, A. (2003). Antipyretic, parasitologic, and immunologic effects of combining sulfadoxine/pyrimethamine with chloroquine or paracetamol for treating uncomplicated Plasmodium falciparum malaria. American Journal of Tropical Medicine and Hygiene, 69(4), 366-371.

Available at: http://ecommons.aku.edu/eastafrica_fhs_mc_pathol/81 
Authors

E. Hugosson, D. Tarimo, M. Troye-Blomberg, S.M. Montgomery, Zul Premji, and A. Bjorkman 


\title{
ANTIPYRETIC, PARASITOLOGIC, AND IMMUNOLOGIC EFFECTS OF COMBINING SULFADOXINE/PYRIMETHAMINE WITH CHLOROQUINE OR PARACETAMOL FOR TREATING UNCOMPLICATED PLASMODIUM FALCIPARUM MALARIA
}

\author{
ELISABETH HUGOSSON, DONATH TARIMO, MARITA TROYE-BLOMBERG, SCOTT M. MONTGOMERY,
} ZUL PREMJI, AND ANDERS BJÖRKMAN

Malaria Research Unit, Division of Infectious Diseases, and Clincial Epidemiology Unit, Department of Medicine, Karolinska Hospital, Karolinska Institutet, Stockholm, Sweden; Department of Immunology, Stockholm University, Stockholm, Sweden; Department of Parasitology/Medical Entomology, Muhimbili University College of Health Sciences, Dar es Salaam, Tanzania

Abstract. Sulfadoxine/pyrimethamine (SP) is increasingly used against malaria in sub-Saharan Africa because of chloroquine resistance. However, chloroquine may have a beneficial antipyretic effect. We therefore compared the combination of SP plus chloroquine, chloroquine alone, SP alone, and SP plus paracetamol in the treatment of uncomplicated Plasmodium falciparum malaria in 175 Tanzanian children (1-4 years old) in a randomized trial. Outcome variables were axillary temperatures every six hours, daily parasitemias, and serum levels of IgG antibodies to $P$. falciparum. Lower mean temperatures (6-48 hours) were achieved with SP plus chloroquine or paracetamol than with SP alone $(P<0.001)$ or chloroquine alone $(P<0.05)$. All three SP-treated groups showed high and similar parasite reduction (0-48 hours), whereas treatment with chloroquine alone was much less effective. Levels of IgG antibodies to $P$. falciparum increased significantly $(P<0.001)$ and similarly in the four treatment groups between days 0,2 . and 3 . Thus, the addition of chloroquine or paracetamol to SP improved the clinical outcome, but did not affect the parasitologic response or antibody production.

\section{INTRODUCTION}

Despite partial resistance of Plasmodium falciparum to chloroquine in sub-Saharan Africa, malaria is still commonly treated with this drug because it is inexpensive and well tolerated. Sulfadoxine/pyrimethamine (SP) is usually the next choice for treatment when chloroquine is no longer used, as it is also affordable, rarely associated with severe side effects, and can be given in a single dose. ${ }^{1,2}$ Several African countries, mainly in eastern Africa, have introduced SP as a firstline treatment. Tanzania has done so recently, but at the time of the present study, chloroquine was still the first-line treatment. Some countries are now also considering combination treatment to increase efficacy and potentially reduce or prevent the evolution of resistance to the drugs used.

Despite better parasitologic response after treatment with SP, when compared with chloroquine, the clinical responses may be less rapid. ${ }^{3}$ An optimal treatment regimen may therefore be to combine SP and chloroquine, thus optimizing both the anti-parasitic and clinical effects.

Two earlier studies have compared the clinical efficacy of SP versus SP plus chloroquine. No significant difference in fever clearance time was reported by Darlow and others, ${ }^{4}$ while Bojang and others had a significantly higher number of patients returning to the clinic because of persistent fever or other clinical symptoms in the SP alone group. ${ }^{5}$ No significant difference in parasite reduction was observed between the treatment groups in these studies. ${ }^{4,5}$ A faster resolution of clinical symptoms during chloroquine treatment may be explained by its anti-inflammatory properties. In vitro, chloroquine inhibits the production of several pro-inflammatory cytokines, believed to contribute to the pathogenesis and clinical manifestations of malaria infection and rheumatoid arthritis, diseases commonly treated with chloroquine. ${ }^{6-12}$ However, the anti-inflammatory effects of chloroquine may also potentially interfere with natural anti-parasitic immune responses.

Paracetamol is a commonly used antipyretic in children and is often combined with SP. However, paracetamol and other antipyretics have been recommended to be used with caution during $P$. falciparum malaria because of alleged poor antipyretic effects and delayed parasite clearance. ${ }^{13,14}$

We wanted to conduct a more comprehensive study than those previously performed, including more frequent recording of temperature and correlating the anti-parasitic effect directly with the antipyretic effect of chloroquine. We also wanted to study a possible effect on the humoral immune response by chloroquine and paracetamol elicited by infection with $P$. falciparum.

This study was therefore designed to compare four treatment regimens all presently in use in Africa, i.e., SP alone, SP in combination with either chloroquine or paracetamol, and chloroquine alone. The outcome variables were fever, parasitemia, and production of $\mathrm{IgG}$ towards crude $P$. falciparum antigens.

\section{METHODS}

Study area. The study was conducted in the Kibaha District Hospital $40 \mathrm{~km}$ northwest of Dar es Salaam, Tanzania. The area is holoendemic for malaria with largely perennial transmission of mainly $P$. falciparum.

Study patients. Children between 12 and 59 months of age attending the primary health care unit of the hospital between July 3 and August 20, 1998 were included in the study if they had uncomplicated mono-infection with $P$. falciparum, a parasite density of $2,000-250,000$ parasites/ $\mu \mathrm{L}$, an axillary temperature of $37.5-40.0^{\circ} \mathrm{C}$, no history of drug intake in the last two weeks, and no history of hypersensitivity drug reactions. Children were not eligible for the study if they had severe malaria as defined by altered level of consciousness, convulsions, prostration, circulatory shock or respiratory distress, hyperparasitemia ( $>250,000$ parasites/ $\mu \mathrm{L})$, or severe anemia (hemoglobin level $<50 \mathrm{~g} / \mathrm{L}$ ). Children with signs of coexisting diseases were also excluded. The guardians of the children satisfying the inclusion criteria were asked for their informed consent for the children to be enrolled into the study. Ethical clearance was obtained from the Ministry of 
Health of Tanzania and the clinical research committee of the Karolinska Institutet (Stockholm, Sweden).

Drug administration. The patients were given serial numbers when attending the primary health care unit, before screening of parasitemia and fever. When admitted to the hospital they were consecutively divided into either of four treatment groups according to a predetermined schedule: 1) chloroquine alone, 2) SP alone, 3) SP plus chloroquine and 4) SP plus paracetamol. More patients were allocated to the group receiving chloroquine alone to balance the two groups receiving SP alone and SP plus paracetamol. Therefore, on each day of enrollment it was decided by a toss of a coin if an additional category 5 for chloroquine alone would be added in the schedule preceding category 1 . The treating physician was not involved in allocating unique identification numbers to the patients deciding the order of admittance into the study. When following the treatment schedule, he could therefore not influence which patient would be allocated to each treatment group. The three drugs were given as follows: 1) chloroquine phosphate (F. Hoffman-La Roche, Basel, Switzerland), $10+10+5 \mathrm{mg}$ base $/ \mathrm{kg}$ of body weight over a period of three days; 2) Fansidar ${ }^{\circledR}$ (F. Hoffman-La Roche), a single dose of $1.25 \mathrm{mg}$ of pyrimethamine and $25 \mathrm{mg}$ of sulfadoxine $/ \mathrm{kg}$ of body weight; and 3) paracetamol, $15 \mathrm{mg} / \mathrm{kg}$ of body weight every eight hours for 72 hours. The children and their guardians were not told what treatment arm they were in. To equalize the number of occasions of tablet intake for the SP- and chloroquine-treated children, each patient also received one tablet of vitamin $\mathrm{C}$ per day for three days. All treatments started at 3:00-4:00 PM on the day of admission and were given under close supervision by the research team. No antipyretic drugs were given to the children apart from the treatment schedule defined. Mechanical antipyresis (tepid sponging and electric fanning) was considered during episodes of high fever $\left(>39^{\circ} \mathrm{C}\right)$.

Assessment of drug efficacy. The primary outcome in the study was temperature at 48 hours and the main secondary outcome was parasite clearance after 72 hours. The children remained in the ward for three days. Axillary body temperature was measured with a digital thermometer on admission, and then every six hours for 72 hours, by the hospital nurses. Parasite species were assessed by Giemsa-stained thin blood smears on admission and parasite densities by thick blood smears daily for four days. The parasite densities were estimated by counting asexual parasites per 200 leukocytes in the thick smear and expressed as number of parasites per microliter of blood, assuming an average number of 8,000 leukocytes/ $\mu \mathrm{L}$ of blood. This was performed by a skilled laboratory assistant. Measures of temperature and counting of parasites were not influenced by knowledge of which anti-malarial treatment that was given to the patient. The need for a double-blinded trial was therefore not considered necessary. A selected number of the patients in this study were previously studied for parasite densities and fever survival time. ${ }^{15}$

Treatment failures. A second-line treatment with quinine (orally or intravenously) was administered if repeated vomiting, signs of clinical deterioration, and/or severe malaria occurred. A second-line treatment with SP was to be given to children treated with chloroquine alone if 1) the axillary temperature was $\geq 37.5^{\circ} \mathrm{C}$ at 48 hours together with increased parasitemia compared with day 0,2 ) the axillary temperature was $\geq 37.5^{\circ} \mathrm{C}$ at 72 hours together with detectable parasit- emia, and 3) the parasitemia at 72 hours was $>25 \%$ of the value at day 0 . In case of treatment failure, data from the patient were included in the analysis up to the time of change of treatment.

Determination of IgG levels. Blood plasma samples for analysis of IgG levels were collected at admission and after 48 and 72 hours. Of 169 patients who participated in the study until 72 hours, 92 were randomly selected for IgG analyses by drawing of lots within each treatment group. Anti-malarial IgG levels were determined as previously described. ${ }^{16}$ Briefly, enzyme-linked immunosorbent assay plates were coated with $50 \mu \mathrm{L}$ of Percoll fractionated and lysed parasite cultures $(10 \mu \mathrm{g} / \mathrm{mL})$ per well. The test sera were diluted 1:1,000 and applied in duplicate. Bound IgG was assayed with alkaline phosphatase-conjugated goat anti-human IgG antibodies (Mabtech, Stockholm, Sweden). Phosphatase substrate (Sigma, St. Louis, MO) was applied and the optical density was read at $450 \mathrm{~nm}$. The $\mathrm{IgG}$ concentrations were calculated from standard curves obtained by coating with goat anti-human IgG (Jackson ImmunoResearch Laboratories, Inc., West Grove, PA), incubating with serial dilutions of recombinant human IgG (Jackson ImmunoResearch Laboratories, Inc.), and assayed as described earlier in this report. The detection limit was set on the linear part of the standard curve and above the background values of buffer controls.

Statistical analysis. Temperature data were analyzed by analysis of variance (ANOVA) repeated measurements design with the independent factors time and treatment. Post hoc comparisons were performed by planned comparison with a $t$-test. The $t$-test was also used when comparing differences in temperature between 18 and 24 hours within each treatment group. The individuals' mean temperatures over time (6-24, 30-48, and 6-48 hours) were analyzed by ANOVA with one independent factor (treatment) at four levels. Post hoc comparisons were done by the Tukey test. The mean temperatures of individuals over time (6-24, 30-48, and 6-48 hours) were also analyzed by multiple regression and conditional change modeling, adjusting for initial temperature, parasitemia, age, and sex as covariables.

All parasite data were $\log$ transformed $\left(\log _{\mathrm{e}}(\mathrm{x}+1)\right)$. Analyses were performed by ANOVA with one independent factor (treatment) at four levels with respect to differences in parasitemia from $0-24$ and $0-48$ hours. Post hoc pairwise comparisons were made by the Tukey test. Differences in parasite reduction from $0-24$ and $0-48$ hours were also analyzed using multiple regression and conditional change modeling, adjusting for initial parasitemia, temperature, age, and sex as co-variables.

The IgG data were $\log$ transformed $\left(\log _{\mathrm{e}}\right)$ and differences between time points $(0,48$, and 72 hours) were analyzed using Wilcoxon matched pairs. Differences in IgG levels between treatment groups were estimated using multiple linear regression with $\log \mathrm{IgG}$ at 48 and 72 hours as dependent variables and treatment as an independent variable with simultaneous adjustment for $\log _{\mathrm{e}} \operatorname{IgG}$ time 0 , age, sex, and parasitemia time 0 . Correlations between changes in temperature and changes in parasitemia from 0 to 48 hours were estimated by a Spearman rank order coefficient. All statistical tests were two sided with significance levels of 0.05 . 


\section{RESULTS}

A total of 175 patients were enrolled in the study. The demographic, clinical, and laboratory data of the four treatment groups are shown in Table 1 . All children eventually recovered, but early treatment failure was recorded in 10 of them, who therefore had to be treated with a second-line treatment. Treatment failure was observed in six children after 48 hours and in another four after 72 hours. Nine children belonged to the group treated with chloroquine alone, whereas one child with treatment failure at 48 hours belonged to the group treated with SP plus chloroquine.

The temperature profiles (0-72 hours) were analyzed by ANOVA repeated measurements design. Different profiles were found between children receiving SP alone and all the other groups, i.e., versus chloroquine $(P=0.003)$, SP plus chloroquine $(P<0.001)$, and SP plus paracetamol $(P<0.001)$. The mean temperatures during the $0-48$-hour time period in the four treatment groups are shown in Figure 1. After 72 hours of treatment, the mean (minimum-maximun) temperatures were as follows in the four groups: $\mathrm{SP}$ alone $=36.2^{\circ} \mathrm{C}$ $\left(35.5-37.0^{\circ} \mathrm{C}\right)$, chloroquine alone $=36.3^{\circ} \mathrm{C}\left(35.1-38.5^{\circ} \mathrm{C}\right), \mathrm{SP}$ plus chloroquine $=36.2^{\circ} \mathrm{C}\left(35.6-36.8^{\circ} \mathrm{C}\right)$, and SP plus paracetamol $36.4^{\circ} \mathrm{C}\left(35.8-37.2^{\circ} \mathrm{C}\right)$. The temperatures increased temporarily between 18 and 24 hours in each of the two groups receiving chloroquine given at 0 and 24 hours $(P<$ 0.007 , by $t$-test) (Figure 1$)$. There were no significant differences between these time points in the other groups.

There was a tendency that SP plus paracetamol gave the best early reduction in fever from 0 to 6 hours. This reached statistical significance when compared with SP or chloroquine alone $(P<0.01)$, but not when compared with SP plus chloroquine $(P=0.3)$.

The mean temperature during the first day (6-24 hours), recorded as area under the curve, was highest in the children treated with SP alone $(P<0.001)$ (Table 2$)$. As the mean temperature in the SP alone group started to decrease, the difference in comparison with the chloroquine alone group decreased and was not significant during the period of 30-48 hours $(P=0.4)$. Chloroquine alone exhibited similar reduction in fever as SP plus paracetamol during the first 24 hours of treatment, but not during the next day (30-48 hours) $(P=$ $0.03)$. Combining chloroquine and SP provided lower temperatures compared with chloroquine alone during both days (6-24 hours; $P=0.03$ and $30-48$ hours; $P<0.001$ ). No significant differences were shown when comparing SP plus chloroquine with SP plus paracetamol during the two-day period. Multiple regression with conditional change modeling adjusting for age, sex, initial temperature, and parasitemia did not change the levels of significance in the analyses of mean temperatures, other than enhancing the differences between the chloroquine alone group and those treated with SP plus chloroquine or SP plus paracetamol to $P<0.005$ (adjusted and unadjusted) for all time points except 6-24 hours for SP plus paracetamol $(P=0.03$, adjusted and unadjusted). Differences in baseline characteristics cannot account for the differences seen between the treatment groups.

The effects of the four treatments on parasite prevalences and densities are shown in Table 3 and Figure 2, respectively. In the chloroquine-treated group, only half (47\%) of the patients were parasite free after 72 hours compared with $87 \%$ in the three SP-treated groups combined. Similarly, whereas the median density was reduced from 24,880 to 1,720 parasites/ $\mu \mathrm{L}$ after 48 hours in the chloroquine-treated children, the median parasitemias were below the detection limit in the other three SP-treated groups. No statistically significant differences in parasite density reduction ( $0-48$ hours) were found between any of the three SP-treated groups by multiple regression $(P$ $>0.09$ ) before and after adjustment for age, sex, initial parasitemia, and temperature. Thus, adding chloroquine to SP did not improve parasite clearance (prevalences or densities). However, parasite reduction between 0 and 24 hours was greater in the group treated with SP plus paracetamol compared with any of the other groups by multiple regression $(P$ $<0.005)$ before and after adjusting for age, sex, initial parasitemia, and temperature.

A weak association $\left(\mathrm{r}^{2}=0.2\right)$ between decrease in temperature and parasitemia was observed only between 0 and 48 hours in the group treated with chloroquine alone (Figure 3). The nine patients with significant reduction of parasite load ( $>$ 3,000-fold, i.e., delta $\log <-8$; Figure 3) showed fever reduction between $1{ }^{\circ} \mathrm{C}$ and $4^{\circ} \mathrm{C}$. In four of six children with an increase in parasitemia, fever increased.

Plasma from 92 children randomly selected within the treatment groups were analyzed for malaria-specific IgG levels. There were general increases in IgG levels by time: day 0 versus day 2 , day 2 versus day 3 , and day 0 versus $3(P<0.001$, by Wilcoxon matched pairs). However, the levels of $\mathrm{IgG}$ before the start of treatment differed somewhat between the treatment groups (Figure 4). The differences were taken into account when comparing the IgG levels for the different treatment groups at days 2 and 3. After adjusting for $\mathrm{IgG}$ levels at day 0 , there were no significant differences between the treatment groups in IgG levels on days 2 or 3 .

\section{DISCUSSION}

The relatively high rate of early treatment failure within three days ( 9 of $56,16 \%$ ) confirms a relatively high degree of

TABLE 1

Baseline characteristics of the four treatment groups at the time of enrollment*

\begin{tabular}{|c|c|c|c|c|}
\hline Characteristics & Chloroquine & SP & $\mathrm{SP}+$ chloroquine & SP + paracetamol \\
\hline No. of patients & 56 & 41 & 40 & 38 \\
\hline Age (months), mean (range) & $(12-57)$ & $(13-50)$ & $(12-52)$ & $(12-52)$ \\
\hline $\operatorname{Sex}($ male/female $)$ & $29 / 27$ & $21 / 20$ & $18 / 22$ & $27 / 11$ \\
\hline Hemoglobin $(\mathrm{g} / \mathrm{L})$, mean (range) & $(59-129)$ & $(55-131)$ & $(56-123)$ & $(53-123)$ \\
\hline Baseline temperature $\left({ }^{\circ} \mathrm{C}\right)$, mean (range) & $38.5 \quad(37.4-40.0)$ & $(37.6-40.2)$ & $(37.6-40.1)$ & $(37.4-40.0)$ \\
\hline Parasitemia (parasites/ $\mu \mathrm{L})$, median (range) & $24,880(2,600-189,560)$ & $42,240(3,040-212,640)$ & $52,200(2,160-189,600)$ & $56,620(4,160-184,960)$ \\
\hline
\end{tabular}




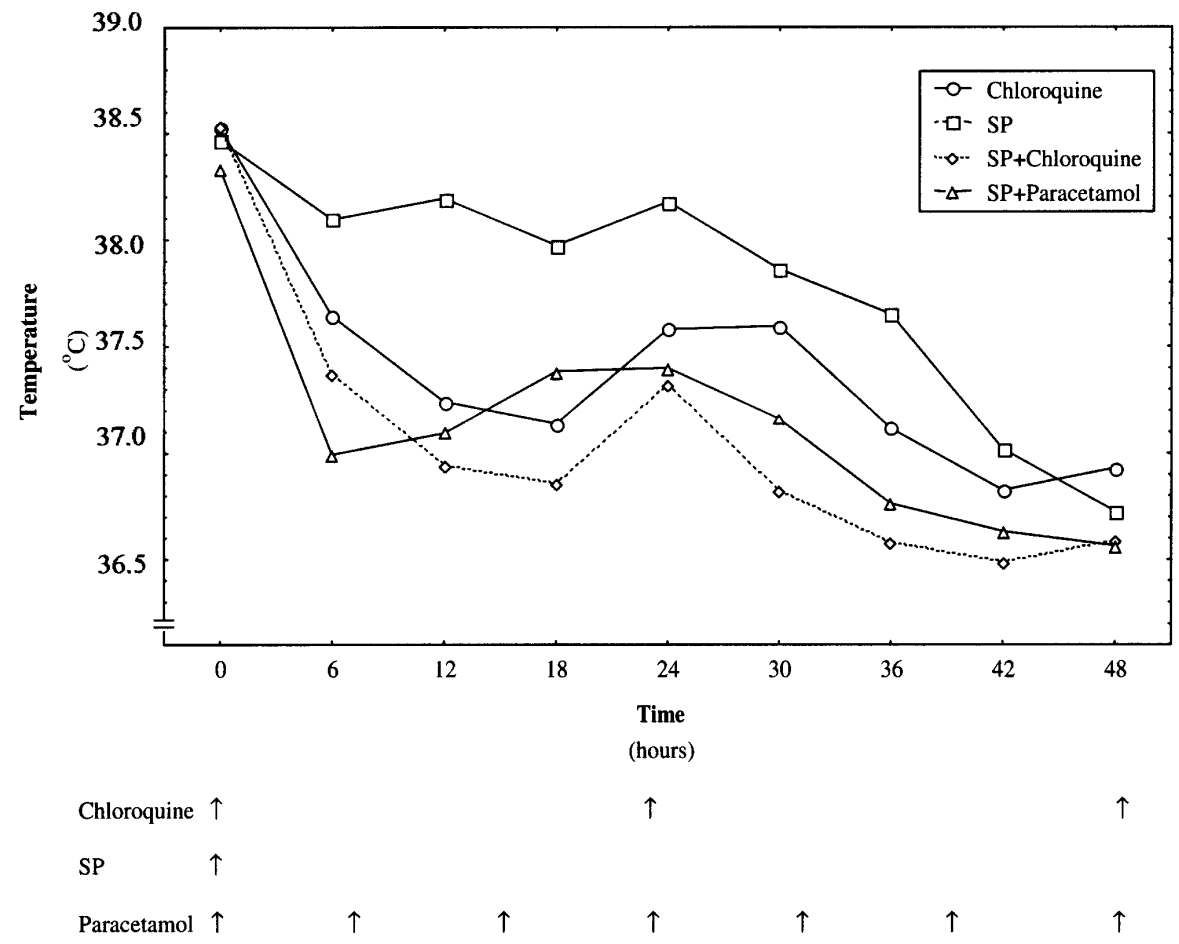

FIGURE 1. Mean axillary temperature in four groups of patients with uncomplicated Plasmodium falciparum malaria. Children in the groups treated with chloroquine alone, sulfadoxine/pyrimethamine (SP) alone, or chloroquine in combination with SP did not receive any antipyretic drugs. Treatment started at 3:00-4:00 PM.

chloroquine resistance in this part of Tanzania. This is in agreement with other in vivo studies in different parts of this country. ${ }^{17,18}$ In contrast, the occurrence of only one early treatment failure by day $3(<1 \%)$ in the SP-treated children suggests a relatively low degree of resistance to SP. The high frequency of treatment failures to SP reported from the Tanga region does not seem to have spread to the Kibaha district. $^{15,19}$

The addition of chloroquine to SP did not improve the parasite clearance of SP alone. This is in agreement with previous studies, ${ }^{4,5}$ although clearance at day 28 turned out to be improved for the combination in one of the studies. ${ }^{5}$ The addition of paracetamol did not negatively affect parasite clearance as was observed in a previous study. ${ }^{13}$ In our study, the reduction of parasite densities during the first 24 hours was most profound in the SP plus paracetamol-treated children. Therefore, we do not believe that paracetamol itself or fever reduction as such should negatively affect the clearance of parasitemia.

The assumed antipyretic effect of chloroquine was con-

TABLE 2

Mean temperature $\left({ }^{\circ} \mathrm{C}\right)$ of malaria patients treated with four different drug regimens*

\begin{tabular}{lccc}
\hline & \multicolumn{3}{c}{ Time (hours) } \\
\cline { 2 - 4 } & $6-24$ & $30-48$ & $6-48$ \\
\hline Chloroquine & 37.4 & 37.1 & 37.3 \\
SP & 38.1 & 37.3 & 37.7 \\
SP + chloroquine & 37.1 & 36.6 & 36.9 \\
SP + paracetamol & 37.2 & 36.8 & 37.0 \\
\hline$*$ SP = sulfadoxine/pyrimethamine. & &
\end{tabular}

firmed because the addition of chloroquine to SP did not improve parasite clearance, but significantly improved fever clearance. Similarly, chloroquine also provided an antipyretic effect comparable to what was achieved with paracetamol. A tendency of increased temperature before the daily chloroquine dosing suggested that the antipyretic effect in both chloroquine-treated groups lasted for less than 24 hours, despite the relatively long early elimination half-life of the drug (approximately 48 hours). ${ }^{20}$ However, the pattern of the fever curve is strikingly similar to what was recorded for quininetreated children. ${ }^{21}$ The time points for low and peak values also correspond with the normal endogenous circadian rhythm (lowest point at approximately 6:00 AM and a peak at approximately 6:00 $\mathrm{PM}) .^{22,23}$ The different pattern detected in the SP alone-treated children implies that this rhythm is disturbed during acute uncomplicated $P$. falciparum malaria treated without antipyretics, and that the antipyretic effect of chloroquine (and possibly quinine) is influenced by what drives the natural 24-hour rhythm.

TABLE 3

Number of patients positive for Plasmodium falciparum parasites after 48 and 72 hours of treatment*

\begin{tabular}{lccccc}
\hline & \multicolumn{2}{c}{48 hours } & & \multicolumn{2}{c}{72 hours } \\
\cline { 2 - 3 } \cline { 5 - 6 } & No. & $\%$ & & No. & $\%$ \\
\hline Chloroquine & $47 / 56$ & 84 & & $27 / 51$ & 53 \\
SP & $13 / 41$ & 32 & & $5 / 41$ & 12 \\
SP + chloroquine & $18 / 40$ & 45 & $6 / 39$ & 15 \\
SP + paracetamol & $17 / 38$ & 45 & $4 / 38$ & 11 \\
Total & $95 / 175$ & 54 & & $42 / 169$ & 25 \\
\hline$*$ *S = sulfadoxine/pyrimethamine. & & &
\end{tabular}




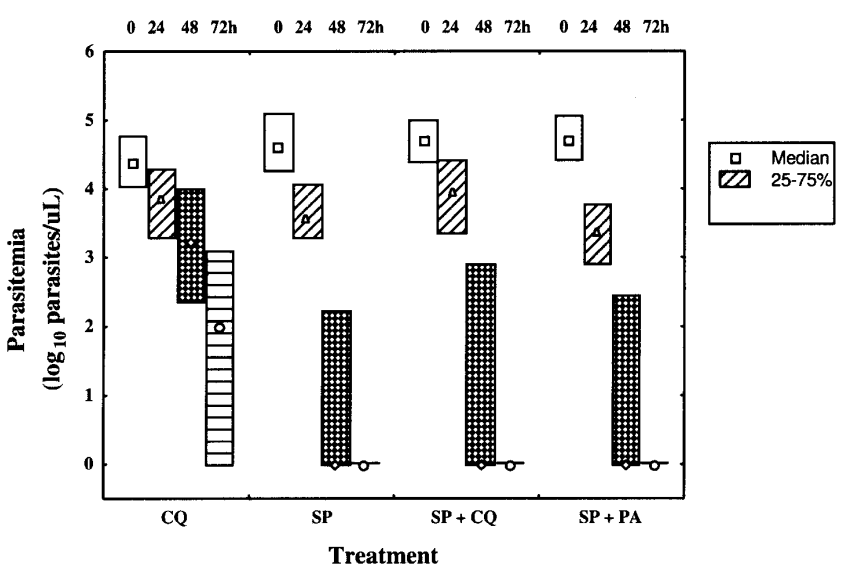

FIgURe 2. Parasite densities (medians and distributions) in children with uncomplicated Plasmodium falciparum malaria after four different treatment regimens. Blood smears were collected each day at 3:00-4:00 PM before drug administration. $\mathrm{h}=$ hours; $\mathrm{CQ}=$ chloroquine; $\mathrm{SP}=$ sulfadoxine/pyrimethamine; $\mathrm{PA}=$ paracetamol.

Some degree of parasite clearance appeared to be required for the antipyretic effect of chloroquine to be significant, since children treated with chloroquine alone had more fever during the second day compared with those treated with SP plus chloroquine, who had more significant parasite recovery. Furthermore, this was also supported by an association between the reduction in fever and the reduction in parasitemia. The antipyretic effect of chloroquine was weak or negligible in most of the few children with early treatment failure. A greater number of observations would be needed for this to be proved. The relatively slow fever recovery effect by SP treatment alone confirms previous similar preliminary findings. ${ }^{3}$ However, the anti-parasitic effect brings about a reduction of fever during the second day, but an antipyretic drug is still required up to 48 hours if clearance of fever is to be achieved.

There was a general increase in IgG levels to P. falciparum $\mathrm{IgG}$ during treatment, indicating ongoing humoral immune responses during acute infection. No difference in this antibody response could be found between the groups, suggesting

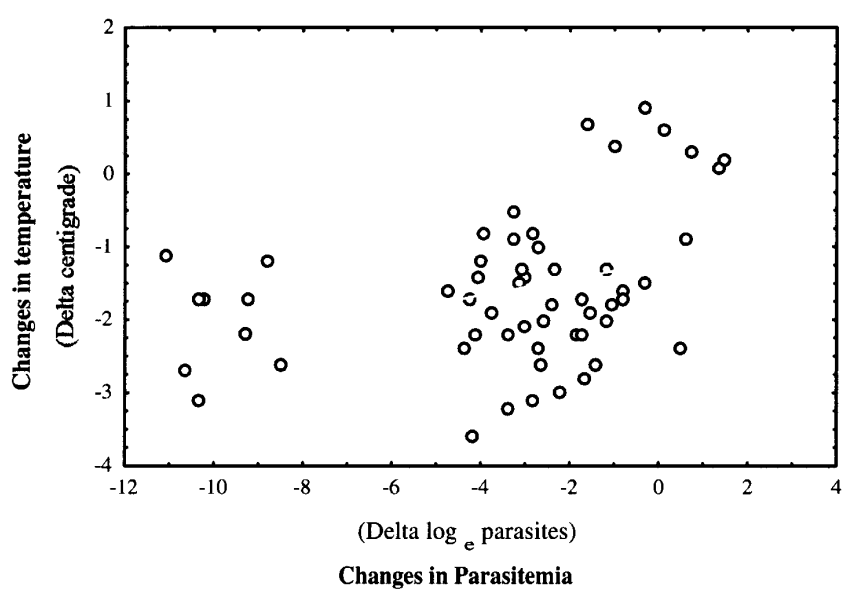

FiguRE 3. Association between changes in temperature and parasite load between 0 and 48 hours for patients with uncomplicated Plasmodium falciparum malaria treated with chloroquine alone

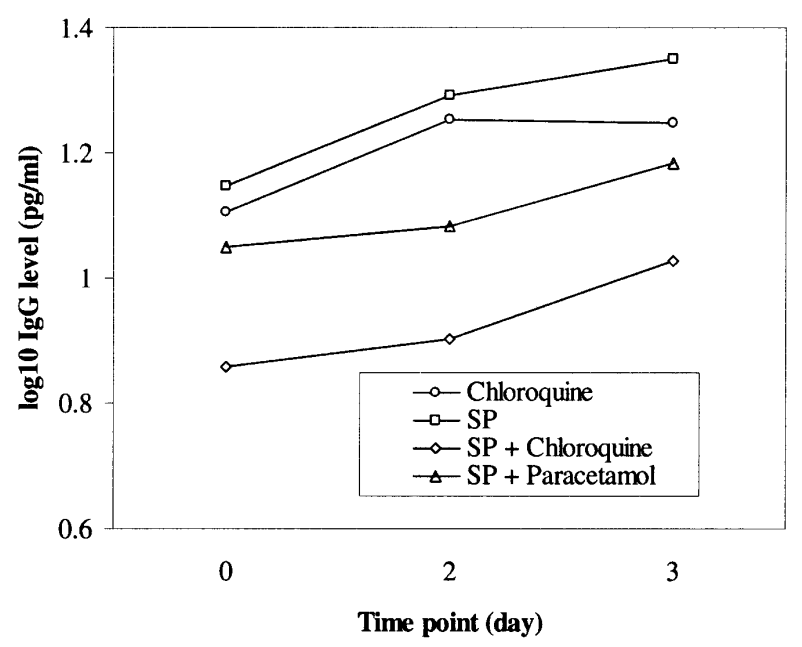

FIGURE 4. Median IgG levels in patients during treatment of uncomplicated Plasmodium falciparum malaria with four different drug regimens. SP = sulfadoxine/pyrimethamine. Twenty-two patients were treated with chloroquine alone, 24 with SP alone, 25 with chloroquine plus SP, and 21 with SP plus paracetamol.

that chloroquine does not appear to influence the humoral immune response despite its anti-inflammatory properties. This supports previous findings that chloroquine prophylaxis does not influence the serologic response to non-live vaccines. $^{24}$

In conclusion, if effective fever clearance is to be achieved, chloroquine, possibly not a full standard anti-malarial treatment dose, or an antipyretic such as paracetamol is necessary as ancillary treatment to SP. The use of these drugs as antipyretics does not seem to interfere with the parasite clearance of SP.

Received March 17, 2003. Accepted for publication July 23, 2003.

Acknowledgments: We thank the children, their families, and the staff at Kibaha Hospital for their participation in this study. Special thanks are given to Fredrick Kalokola, Jeremiah Masunga (deceased), and Anette Sundstedt for laboratory work and organization at the hospital. We are grateful to Margareta Hagstedt and Hedvig Perlmann for technical assistance with the antibody enzyme-linked immunosorbent assay, and Jan Kowalski for statistical expertise. We also thank Amos Y. Massele, Anita Östborn, Ulrika Uddenfeldt, Ib Bygbjerg, and Anita Rønn for their contributions in making the study possible.

Financial support: The work was supported by Swedish International Development Co-operation Agency (Stockholm, Sweden).

Authors' addresses: Elisabeth Hugosson, Malaria Research Unit (M9), Division of Infectious Diseases, Department of Medicine, Karolinska Hospital, 17176 Stockholm, Sweden, Telephone: 46-85177-6739, Fax: 46-8-5177-6740, E-mail:ehugosson@hotmail.com. Donath Tarimo and Zul Premji, Department of Parasitology/Medical Entomology, Muhimbili University College of Health Sciences, Dar es Salaam, Tanzania, Telephone: 255-22-215-1596, Fax: 255-22-2150465, E-mails: dtarimo@muchs.ac.tz and zpremji@muchs.ac.tz. Marita Troye-Blomberg, Department of Immunology, Stockholm University, Stockholm, Sweden, Telephone: 46-8-164-164, Fax: 46-8612-9542 or 46-8-157-356, E-mail: marita@imun2.su.se. Scott M. Montgomery, Clinical Epidemiology Unit, Department of Medicine, Karolinska Hospital, Karolinska Institutet, 17176 Stockholm, Sweden, Telephone: 46-8-5177-9325, Fax: 46-8-5177-9304, E-mail: Scott.Montgomery@medks.ki.se. Anders Björkman, Malaria Research Unit (M9), Division of Infectious Diseases, Department of Medicine, Karolinska Hospital, 17176 Stockholm, Sweden, Telephone: 46-8-5177-1866, Fax: 46-8-5177-6740, E-mail: anders. bjorkman@ks.se. 


\section{REFERENCES}

1. World Health Organisation, 1990. Practical Chemotherapy of Malaria. World Health Organ Tech Rep Ser 805.

2. Sturchler D, Mittelholzer ML, Kerr L, 1993. How frequent are notified severe cutaneous adverse reactions to Fansidar? Drug Saf 8: $160-168$

3. Muller O, van Hensbroek MB, Jaffar S, Drakeley C, Okorie C, Joof D, Pinder M, Greenwood B, 1996. A randomized trial of chloroquine, amodiaquine and pyrimethamine-sulphadoxine in Gambian children with uncomplicated malaria. Trop Med Int Health 1: 124-132.

4. Darlow B, Vrbova H, Gibney S, Jolley D, Stace J, Alpers M, 1982. Sulfadoxine-pyrimethamine for the treatment of acute malaria in children in Papua New Guinea. I. Plasmodium falciparum. Am J Trop Med Hyg 31: 1-9.

5. Bojang KA, Schneider G, Forck S, Obaro SK, Jaffar S, Pinder M, Rowley J, Greenwood BM, 1998. A trial of Fansidar plus chloroquine or Fansidar alone for the treatment of uncomplicated malaria in Gambian children. Trans $R$ Soc Trop Med Hyg 92: 73-76.

6. Ertel W, Morrison MH, Ayala A, Chaudry IH, 1991. Chloroquine attenuates hemorrhagic shock-induced suppression of Kupffer cell antigen presentation and major histocompatibility complex class II antigen expression through blockade of tumor necrosis factor and prostaglandin release. Blood 78: 1781-1788.

7. Landewe RB, Miltenburg AM, Breedveld FC, Daha MR, Dijkmans BA, 1992. Cyclosporine and chloroquine synergistically inhibit the interferon-gamma production by CD4 positive and CD8 positive synovial T cell clones derived from a patient with rheumatoid arthritis. J Rheumatol 19: 1353-1357.

8. Picot S, Peyron F, Donadille A, Vuillez JP, Barbe G, AmbroiseThomas P, 1993. Chloroquine-induced inhibition of the production of TNF, but not of IL-6, is affected by disruption of iron metabolism. Immunology 80: 127-133.

9. van den Borne BE, Dijkmans BA, de Rooij HH, le Cessie S, Verweij CL, 1997. Chloroquine and hydroxychloroquine equally affect tumor necrosis factor-alpha, interleukin 6, and interferon-gamma production by peripheral blood mononuclear cells. J Rheumatol 24: 55-60.

10. Jeong JY, Jue DM, 1997. Chloroquine inhibits processing of tumor necrosis factor in lipopolysaccharide-stimulated RAW 264.7 macrophages. J Immunol 158: 4901-4907.

11. Karres I, Kremer JP, Dietl I, Steckholzer U, Jochum M, Ertel W, 1998. Chloroquine inhibits proinflammatory cytokine release into human whole blood. Am J Physiol 274: R1058-R1064.

12. Hugosson E, Bjorkman A, Troye-Blomberg M, 2002. Chloroquine enhances the number of IL-10 producing cells and the expression of B7-2 and ICAM-1 in in vitro-cultured PBMC. Scand J Immunol 55: 399-408.

13. Brandts CH, Ndjave M, Graninger W, Kremsner PG, 1997. Effect of paracetamol on parasite clearance time in Plasmodium falciparum malaria. Lancet 350: 704-709.

14. Lell B, Sovric M, Schmid D, Luckner D, Herbich K, Long HY, Graninger W, Kremsner PG, 2001. Effect of antipyretic drugs in children with malaria. Clin Infect Dis 32: 838-841.

15. Tarimo DS, Minjas JN, Bygbjerg IC, 2002. Sulfadoxinepyrimethamine monotherapy in Tanzanian children gives rapid parasite clearance but slow fever clearance that is improved by chloroquine in combination therapy. Trop Med Int Health 7: 592-598.

16. Perlmann H, Helmby H, Hagstedt M, Carlson J, Larsson PH, Troye-Blomberg M, Perlmann P, 1994. IgE elevation and IgE anti-malarial antibodies in Plasmodium falciparum malaria: association of high IgE levels with cerebral malaria. Clin Exp Immunol 97: 284-292.

17. Ekvall H, Premji Z, Bjorkman A, 1998. Chloroquine treatment for uncomplicated childhood malaria in an area with drug resistance: early treatment failure aggravates anaemia. Trans $R$ Soc Trop Med Hyg 92: 556-560.

18. Ministry of Health T, 2000. Implementation of a new anti-malaria treatment policy in Tanzania: rationale for change and guide to the process of policy implementation, Task Force Report September 1999. Tanzania Health Res Bull 2: 10-13.

19. Ronn AM, Msangeni HA, Mhina J, Wernsdorfer WH, Bygbjerg IC, 1996. High level of resistance of Plasmodium falciparum to sulfadoxine- pyrimethamine in children in Tanzania. Trans $R$ Soc Trop Med Hyg 90: 179-181.

20. Rombo L, Bjorkman A, Sego E, Ericsson O, 1986. Whole blood concentrations of chloroquine and desethylchloroquine during and after treatment of adult patients infected with Plasmodium vivax, P. ovale or P. malariae. Trans $R$ Soc Trop Med Hyg 80: 763-766.

21. Lell B, Brandts CH, Graninger W, Kremsner PG, 2000. The circadian rhythm of body temperature is preserved during malarial fever. Wien Klin Wochenschr 112: 1014-1015.

22. Ogle W, 1866. On the diurnal variations in the temperature of the human body in health. St. Georgés Hosp Rep 1: 221-245.

23. Conroy RT, Mills JN, 1970. Human circadian rhythms. First edition. London: Churchill.

24. Gyhrs A, Pedersen BK, Bygbjerg I, Henrichsen J, Heron I, Petersen I, Skinhoj P, 1991. The effect of prophylaxis with chloroquine and proguanil on delayed- type hypersensitivity and antibody production following vaccination with diphtheria, tetanus, polio, and pneumococcal vaccines. Am J Trop Med Hyg 45: 613-618. 